\title{
Fatores que influenciam na migração do MPS.BR para o CMMI nas empresas de software brasileiras
}

\author{
Rhavy M. Guedes ${ }^{1,2}$, Rharon M. Guedes ${ }^{2}$, Alexandre L. de Vasconcelos ${ }^{1}$ \\ ${ }^{1}$ Centro de Informática - Universidade Federal de Pernambuco (UFPE) - Recife, PE - \\ Brasil \\ $\{$ rmg3, amlv\}@cin.ufpe.br \\ ${ }^{2}$ Instituto Federal de Educação, Ciência e Tecnologia da Paraíba (IFPB) - Campina \\ Grande, PB - Brasil \\ $\{$ rhavy.guedes, rharon.guedes\} eifpb.edu.br
}

\begin{abstract}
The software industry in Brazil are increasingly investing in software process improvement, being MPS an economically viable model. However, several national companies have migrated their MPS assessment to CMMI. In this context, this research aims to identify the factors that influence the migration of MPS model to CMMI. Were analyzed the lists of companies assessed by MPS and CMMI, also was performed a non-systematic review of the literature and two surveys. It was found evidences about migration from MPS initial levels to CMMI, the factors related to internationalization, and requirement of customers were identified as key motivators.
\end{abstract}

Resumo. A indústria de software no Brasil investe cada vez mais em melhoria dos seus processos, sendo o modelo MPS uma opção economicamente viável. Contudo, muitas empresas nacionais migram sua avaliação do MPS para o CMMI. Neste contexto, esta pesquisa busca identificar os fatores que influenciam a migração do modelo MPS para o CMMI. Foram analisadas as listas das empresas avaliadas pelo MPS e CMMI, também, executada uma revisão não sistemática da literatura e duas pesquisas de campo. Foram encontradas evidências de migração dos níveis iniciais do MPS para o CMMI e os fatores que envolvem a internacionalização e exigência dos clientes foram apontados como motivadores principais.

\section{Introdução}

O crescimento da indústria de software brasileira, assim como as mudanças do setor e o aumento da competitividade motivam as empresas desenvolvedoras de software a implantar a Melhoria no Processo de Software (Software Process Improvement - SPI), além de ser um diferencial de mercado [Softex, 2012a], [Almeida, 2011]. A melhoria no processo de software nas organizações pode aumentar a produtividade, reduzir custos e ainda aumentar a satisfação dos clientes devido à melhora na qualidade dos seus produtos e serviços [Monotoni, 2010], [Fuggetta; 2000], [Gibson et al., 2006], [Travassos e Kalinowski, 2012).

Diferentes abordagens de SPI estão disponíveis no mercado, a citar, ISO/IEC 12207 [ISO, 2008], ISO/IEC 15504 [ISO, 2003], Melhoria do Processo de Software Brasileiro (MPS.BR) [Softex, 2012a] e Capability Maturity Model Integration (CMMI) [SEI, 2010] que devem estar alinhadas com os objetivos de negócio e projetos da organização 
[Macmahon, 2011]. Estudos mostram que as organizações estão propensas a adotar modelos desenvolvidos em sua localidade regional e adaptados à sua realidade e contexto [Unterkalmsteiner, 2012], [Softex, 2012a]. A Avaliação Anual de Desempenho em empresas de software que adotaram o MPS, identificada pela sigla iMPS, apontou que a indústria nacional de software de pequeno e médio porte (PME) está interessada em adotar, inicialmente, o Modelo de Referência definido pelo programa de Melhoria do Processo de Software Brasileiro para Software (MR-MPS-SW) [Travassos, 2012], [Santos et al., 2010].

Apesar das facilidades e ações promovidas pela Associação para Promoção da Excelência do Software Brasileiro (Softex) para adoção do modelo MR-MPS-SW, as empresas brasileiras de software, que se encontram nos níveis iniciais de maturidade desse modelo podem apresentar aderência incompleta aos processos [Almeida, 2011] ou até a migração para os níveis equivalentes ou mais elevados do CMMI para Desenvolvimento (CMMIDEV). A migração do MR-MPS-SW para o CMMI-DEV é considerada quando próximo de expirar o prazo da avaliação do modelo MR-MPS-SW, a organização avalia seu nível de maturidade no CMMI-DEV e não renova o certificado do modelo brasileiro. Em algumas situações, no processo de migração, a organização permanece por um curto prazo certificada nos dois modelos. Além disso, destaca-se que não existem componentes específicos do programa MPS.BR capazes de identificar especificamente quais fatores são responsáveis pelo processo de migração nas empresas que adotaram o modelo, apesar da realização periódica, pela Softex, de pesquisas e publicação de relatórios de informações para acompanhar e evidenciar a variação de desempenho [Travassos, 2013].

Diante deste contexto, verifica-se a necessidade de buscar evidências de fatores que influenciam as organizações a migrarem do modelo de melhoria de processo de software brasileiro, o MR-MPS-SW, para o CMMI-DEV, considerando que os modelos são compatíveis [Weber, 2005b], ou seja, seus processos se assemelham.

\section{Metodologia}

As perguntas de pesquisa, métodos de coleta e análise dos dados propostos nesta pesquisa levam em consideração o guia proposto por Kitchenham e Pfleeger (2008).

\subsection{Questões de pesquisa}

As questões de pesquisa buscaram identificar a existência do fenômeno de migração, sua descrição e classificação. Dessa forma, este trabalho objetivou responder as questões de pesquisa apresentadas no Quadro 1.

\section{Quadro 1 - Questões de pesquisa.}

\begin{tabular}{|l|l|l|}
\hline ID & Questão de pesquisa & Objetivo \\
\hline RQ1 & $\begin{array}{l}\text { Há migração da avaliação para o } \\
\text { CMMI-DEV de empresas sediadas no } \\
\text { Brasil e avaliadas no MR-MPS-SW? }\end{array}$ & $\begin{array}{l}\text { Evidenciar a existência de migração } \\
\text { entre o modelo MR-MPS-SW e o } \\
\text { CMMI-DEV. }\end{array}$ \\
\hline RQ2 & $\begin{array}{l}\text { Caso haja migração, qual o perfil das } \\
\text { empresas que migraram do modelo } \\
\text { MR-MPS-SW para o modelo CMMI- } \\
\text { DEV? }\end{array}$ & $\begin{array}{l}\text { Identificar quais são as } \\
\text { características das empresas que } \\
\text { realizaram a migração. }\end{array}$ \\
\hline
\end{tabular}




\begin{tabular}{|l|l|l|}
\hline RQ3 & $\begin{array}{l}\text { Quais fatores influenciam na migração } \\
\text { de empresas certificadas no modelo } \\
\text { MPS para o modelo CMMI? }\end{array}$ & $\begin{array}{l}\text { Identificar quais fatores estão } \\
\text { envolvidos na migração de um } \\
\text { modelo para o outro. }\end{array}$ \\
\hline
\end{tabular}

\subsection{Métodos de coleta}

Para coleta de dados foram utilizados três métodos: extração dos dados da lista das empresas avaliadas pelo MPS.BR e CMMI, revisão não sistemática da literatura e duas pesquisas de campo. A análise da base de dados das certificações do MPS.BR e CMMI identificou inicialmente as empresas que realizaram o processo de migração. A revisão da literatura foi o meio utilizado para identificar, avaliar e interpretar as pesquisas relevantes disponíveis que estão relacionadas à pergunta de pesquisa [Kitchenham e Pfleeger, 2008] desse trabalho. A primeira pesquisa de campo (survey) foi realizada junto ao grupo de avaliadores e implementadores do MPS para identificar outras empresas que realizaram a migração e levantar alguns fatores envolvidos no processo. Já a segunda pesquisa de campo foi aplicada nas empresas que realizaram a migração entre os modelos para confirmar os fatores que influenciaram para o processo de mudança de modelos.

A pesquisa extraiu inicialmente os dados das avaliações oferecidos pela Softex e CMMI Institute. As informações obtidas da literatura e da pesquisa de campo realizada junto ao grupo de implementadores e avaliadores do MPS foram analisadas através da síntese interpretativa dos dados [Noblit e Hare, 1988]. Durante a realização da pesquisa de campo foram utilizadas ferramentas inquisitivas [Singer, Sim e Lethbridge, 2008] - questionário e entrevista - que permitiram o entendimento geral sobre o processo analisado pela pesquisa.

Os dados quantitativos coletados foram analisados através da estatística descritiva e aplicação de testes não-paramétricos, já os dados qualitativos foram analisados através da metodologia definida pela teoria fundamentada em dados (Grounded Theory) proposta por Strauss e Corbin (1998).

Neste trabalho o processo de migração é definido como variável dependente, que é afetada pelas variáveis independentes [Marconi e Lakatos, 2008], formadas pelos fatores que influenciam o processo de migração entre os modelos.

\subsubsection{Levantamento de indícios de migração entre modelos}

Os órgãos reguladores dos modelos CMMI-DEV e MR-MPS-SW disponibilizam na internet as informações referentes ao processo de avaliação de todas as empresas de software certificadas. Atualmente, o CMMI Institute, após o desmembramento com o Software Engineering Institute (SEI), coordena a lista das empresas a nível mundial. Nessa lista, as empresas estão classificadas de acordo com a versão do modelo adotada, nível de maturidade, ano da certificação, avaliadores, prazo de vigência da certificação, nome da unidade organizacional avaliada e seu país de origem. De maneira similar, a Softex disponibiliza os dados das avaliações realizadas desde a implantação do programa MPS.BR no Brasil. As certificações estão agrupadas por ano e vigência, compondo-se pelo resultado da avaliação e por uma declaração de validade do resultado, o qual possui informações gerais sobre a unidade organizacional, data de realização e validade da avaliação, avaliadores, versão do MPS adotada, nível de maturidade e áreas de processos atendidas. Diante destas informações, foram aplicados filtros capazes de identificar as empresas que possuíam certificações válidas ou expiradas no MPS, e que estivessem com 
certificações recentes e dentro do prazo de validade no CMMI, caracterizando assim a migração.

\subsubsection{Levantamento dos fatores da literatura}

A revisão assistemática da literatura iniciou-se pela busca de trabalhos publicados em jornais, simpósios, conferências e workshops nacionais e internacionais que possuíam a instrumentação metodológica e os objetivos correlacionados com a pesquisa descrita nesse documento. A revisão assistemática da literatura permitiu maior flexibilidade na avaliação dos documentos, sem definição de um protocolo rígido. No entanto, o processo assistemático identificou, selecionou e avaliou criticamente as pesquisas mais relevantes. Nelas foram identificados alguns fatores críticos, mais relevantes e recorrentes, envolvidos no sucesso e continuidade de SPI em organizações de software. A seleção final dos fatores levantados na literatura passou pela análise de três membros experientes na comunidade de SPI [Dyba, 2000]. Cada fator foi classificado em: adequado, inapropriado e indicador. Os adequados foram adicionados à lista de fatores utilizada na aplicação da pesquisa de campo junto às empresas que realizaram a migração.

\subsubsection{Aplicação do questionário ao grupo de implementadores e avaliadores do MPS}

Realizou-se uma pesquisa de campo exploratória, de caráter quantitativo e qualitativo, que teve como instrumento de coleta de dados um questionário, enviado por e-mail para o grupo de avaliadores e implementadores do MPS. O questionário (Quadro 2) composto por questões abertas, foi aplicado com o objetivo encontrar respostas para as questões de pesquisa RQ2 e RQ3. Os dados extraídos foram analisados por meio do método de síntese interpretativa dos dados [Noblit e Hare, 1988], que extraiu os códigos e conceitos existentes.

Quadro 2 - Questionário da pesquisa de campo para os avaliadores e implementadores do MPS.

\begin{tabular}{|l|l|}
\hline ID & Pergunta do questionário \\
\hline Q1 & $\begin{array}{l}\text { Você conhece alguma empresa brasileira certificada pelo modelo MR-MPS-SW } \\
\text { que adotou, posteriormente, em níveis mais altos, o CMMI-DEV? Em quantas } \\
\text { empresas é possível identificar a ocorrência? }\end{array}$ \\
\hline Q2 & O que motiva as empresas a se certificarem nos dois modelos? \\
\hline Q3 & $\begin{array}{l}\text { Qual a região do país em que a adoção de outro modelo ocorre com mais } \\
\text { frequência? }\end{array}$ \\
\hline
\end{tabular}

As informações coletadas na revisão da literatura e na aplicação do questionário foram correlacionadas para definir o perfil das empresas que realizaram ou estavam no processo de migração e identificar o conjunto dos fatores envolvidos na migração entre os modelos. Os novos fatores identificados nessa etapa foram adicionados à lista de fatores inicial, extraída da revisão da literatura. Essa lista consolidada serviu como base para a investigação aplicada às empresas.

\subsubsection{Pesquisa de campo transversal nas empresas identificadas na migração}

A pesquisa aplicou um questionário fechado e uma entrevista semiestruturada para o conjunto de empresas de software que realizaram a migração do MR-MPS-SW para o 
CMMI-DEV. As empresas selecionaram um grupo de voluntários (self-selecting) para participar da pesquisa, onde foi preconizado que cada participante deveria ter pelo menos um dos perfis: desenvolvedor (desenvolvedores, analista de negócio ou diretor técnico), gerente (gerentes de qualidade e projeto ou líderes de projeto) e gestor (gerentes seniores ou diretores).

A aplicação do questionário objetivou responder às questões de pesquisa presentes no Quadro 1 e também abordar questões referentes aos fatores que influenciaram a migração. Os blocos das questões foram organizados em: I. Identificação do participante; II. Identificação da organização; III. Identificação do contexto organizacional; IV. Identificação dos problemas relacionados ao modelo MPS que levaram à migração. As questões dos blocos III e IV verificaram o grau de influência dos fatores envolvidos na migração por meio da escala Likert [Likert, 1932]: Discordo fortemente, Discordo, Neutro, Concordo, Concordo fortemente. O Ranking Médio (RM) foi utilizado para analisar os itens da escala Likert respondidos em cada questão [Oliveira, 2005], para isso, substituiu-se a escala qualitativa por respostas quantitativas: Discordo fortemente (1), Discordo (2), Neutro (3), Concordo (4) e Concordo fortemente (5).

Após a condução do questionário, foi aplicada uma entrevista semiestruturada dividida nas seguintes etapas: I. Caracterização do entrevistado; II. Caracterização do processo de melhoria implantado baseado no MPS; III. Verificação da migração e seus motivadores.

Os dados quantitativos coletados do questionário e entrevista foram tratados por meio de métodos da estatística descritiva e testes não-paramétricos. Correlações entre as questões do questionário e da entrevista foram estabelecidas com objetivo de analisar precisamente o posicionamento dos participantes e remover distorções em suas opiniões.

A Teoria Fundamentada em Dados, do inglês, Grounded Theory (GT) [Glaser e Strauss, 1967] foi utilizada para a transcrição e tabulação das entrevistas, criação dos códigos e seus relacionamentos. Da GT foram aplicadas as etapas de codificação aberta e axial com o objetivo de verificar a influência dos fatores.

\section{Resultados}

A lista de avaliações disponibilizada pela Softex em 01 de janeiro de 2013 era composta por 397 empresas brasileiras, das quais 190 ainda se encontravam com o prazo de avaliações vigentes no MPS. No mesmo período, a lista de avaliação disponibilizada pelo CMMI Institute era composta por 3833 empresas, das quais 256 encontravam-se com a avaliação vencida. O cruzamento dos resultados das avaliações das listas do MPS e do CMMI apontou a existência de sete organizações que realizaram a migração entre os anos de 2012 e 2013. A divisão do grau de maturidade no MR-MPS-SW das empresas era: 2 no nível G, 3 no nível F, 1 no nível $\mathrm{C}$ e outra no $\mathrm{A}$. Esse resultado indica que o processo de migração se concentrou em empresas avaliadas nos níveis iniciais de maturidade do MR-MPS-SW. Além disso, surgiram evidências de organizações com a certificação do MR-MPS-SW prestes a expirar e iniciando o processo de certificação no CMMI.

\subsection{Identificação preliminar dos fatores de sucesso em SPI}

Foram correlacionados 27 trabalhos que abordavam os fatores que influenciam o sucesso de SPI com ênfase nas perspectivas de implantação, na análise de fatores críticos de sucesso e de continuidade dos modelos de melhoria no processo de software existentes. 
Foi possível identificar uma lista de fatores de sucesso (Quadro 3) que influenciaram diretamente na adoção, implantação, avaliação e continuidade de SPI.

4.2 Visão dos implementadores e avaliadores do MPS sobre o processo de migração do MR-MPS-SW para o CMMI-DEV

A pesquisa de campo realizada junto ao grupo de implementadores e avaliadores do MPS obteve o retorno de 11 questionários que evidenciaram a migração do MR-MPS-SW para o CMMI-DEV em empresas brasileiras. Foram apontadas pelo grupo mais 10 empresas sediadas no Brasil que realizaram a migração entre os modelos. Desse total, apenas quatro foram enquadradas no processo de migração definido por este trabalho. As respostas obtidas dos questionários indicaram que o processo de migração se concentrou nas regiões Sul, Sudeste, Centro-Oeste (Brasília) e Nordeste (Pernambuco). Também foram identificados seis novos fatores que estavam ligados mais próximos ao processo de migração do modelo MR-MPS-SW para o CMMI-DEV.

A consolidação dos fatores (Quadro 3) reúne e identifica a origem dos fatores encontrados na revisão da literatura e na análise dos dados originários a aplicação do questionário com o grupo de implementadores e avaliadores do MPS. Os fatores consolidados auxiliaram na execução da pesquisa de campo aplicada junto às empresas, pois serviram como base para a construção do questionário e da entrevista utilizados.

Quadro 3 - Consolidação dos fatores.

\begin{tabular}{|c|c|c|c|}
\hline ID & Fator & Literatura & $\begin{array}{l}\text { Grupo } \\
\text { MPS }\end{array}$ \\
\hline F1 & $\begin{array}{l}\text { Estratégia de implantação da melhoria do processo } \\
\text { de software. }\end{array}$ & • & \\
\hline $\mathrm{F} 2$ & $\begin{array}{l}\text { Conciliação dos objetivos do modelo com os } \\
\text { interesses da organização. }\end{array}$ & $\bullet$ & \\
\hline F3 & $\begin{array}{l}\text { Competência dos membros da organização de } \\
\text { software. }\end{array}$ & $\bullet$ & \\
\hline $\mathrm{F} 4$ & $\begin{array}{l}\text { Respeito da consultoria pela organização de } \\
\text { software }\end{array}$ & $\bullet$ & \\
\hline F5 & $\begin{array}{l}\text { Motivação e satisfação dos membros da } \\
\text { organização. }\end{array}$ & • & \\
\hline F6 & Metodologia formal da implantação. & $\bullet$ & \\
\hline F7 & Monitorar e controlar os processos da organização & $\bullet$ & \\
\hline F8 & Falta de competência da consultoria especializada. & $\bullet$ & \\
\hline F9 & Nível de comprometimento da equipe. & $\bullet$ & \\
\hline F10 & $\begin{array}{l}\text { Disputas políticas dentro da organização a ser } \\
\text { avaliada. }\end{array}$ & $\bullet$ & $\bullet$ \\
\hline F11 & Interesse apenas na certificação. & $\bullet$ & $\bullet$ \\
\hline F12 & $\begin{array}{l}\text { Compatibilidade entre os modelos MR-MPS- } \\
\text { SWMPS e CMMI-DEV. }\end{array}$ & • & $\bullet$ \\
\hline
\end{tabular}




\begin{tabular}{|l|l|c|c|}
\hline ID & Fator & Literatura & $\begin{array}{c}\text { Grupo } \\
\text { MPS }\end{array}$ \\
\hline F13 & Valorização da Empresa. & & $\bullet$ \\
\hline F14 & Exigência do Cliente. & & $\bullet$ \\
\hline F15 & Pressão comercial (Concorrência e Competição). & & $\bullet$ \\
\hline F16 & Internacionalização da organização. & & $\bullet$ \\
\hline F17 & Reconhecimento do modelo CMMI no exterior. & & $\bullet$ \\
\hline F18 & Fomento para implantação do MR-MPS-SW. & & $\bullet$ \\
\hline
\end{tabular}

4.3 Identificação dos fatores que influenciaram na migração das empresas de software

A pesquisa de campo foi realizada junto a 7 empresas de software, identificadas no processo de migração. Receberam o questionário e a entrevista um total de 12 indivíduos: sete gerentes (qualidade ou projeto) e cinco analistas (sistemas ou requisitos). Os participantes com o perfil de gerente e desenvolvedor relataram que possuíam pelos menos uma experiência de implantação e execução dos processos previstos no MR-MPSSW ou CMMI-DEV. Além disso, 75\% dos participantes possuíam experiência na implantação do CMMI. Parte das informações iniciais sobre as empresas serviram como base da questão levantada pela RQ2 (Quadro 1).

As questões Q15 a Q35 do questionário avaliaram o grau de influência dos fatores envolvidos na migração. A Tabela 1 apresenta os valores de RM para as questões da Seção III e da Seção IV do questionário (Q15 a Q35).

Tabela 1 - Ranking médio (RM) das respostas do questionário (Q15 a Q35).

\begin{tabular}{|l|l|l|}
\hline ID & Questão & RM \\
\hline Q15 & A organização pretende expandir o mercado para outros países. & 3,84 \\
\hline Q16 & $\begin{array}{l}\text { A organização implantou simultaneamente o MR-MPS-SW e o } \\
\text { CMMI-DEV com o objetivo de aumentar as oportunidades de negócio } \\
\text { dentro e fora do Brasil. }\end{array}$ & 4,09 \\
\hline Q17 & $\begin{array}{l}\text { A organização migrou do MR-MPS-SW para o CMMI-DEV como os } \\
\text { objetivos de aumentar as oportunidades de negócio dentro e fora do } \\
\text { Brasil. }\end{array}$ & 3,50 \\
\hline Q18 & $\begin{array}{l}\text { Houve discordâncias na escolha do MR-MPS-SW para a implantação } \\
\text { da melhoria de processo de software na organização. }\end{array}$ & 2,59 \\
\hline Q19 & $\begin{array}{l}\text { Alguns clientes da organização exigem que o processo de melhoria de } \\
\text { software seja certificado através do CMMI, pois é reconhecido no } \\
\text { exterior. }\end{array}$ & 3,00 \\
\hline Q20 & $\begin{array}{l}\text { A organização considera que o investimento em níveis mais altos do } \\
\text { MR-MPS-SW é equivalente ao CMMI-DEV, por isso opta pelo } \\
\text { modelo internacional. }\end{array}$ & 3,67 \\
\hline Q21 & $\begin{array}{l}\text { A mudança no modelo de gerência dos processos da organização } \\
\text { influenciou na migração do MR-MPS-SW para o CMMI. }\end{array}$ & 2,34 \\
\hline
\end{tabular}




\begin{tabular}{|c|c|c|}
\hline ID & Questão & $\mathbf{R M}$ \\
\hline Q22 & $\begin{array}{l}\text { A mudança dos gestores da organização influenciou na migração do } \\
\text { MR-MPS-SW para o CMMI-DEV. }\end{array}$ & 2,09 \\
\hline Q23 & $\begin{array}{l}\text { A mudança para o CMMI foi influenciada pela diminuição da demanda } \\
\text { pelos produtos ou serviços da organização. }\end{array}$ & 2,09 \\
\hline Q24 & $\begin{array}{l}\text { A rotatividade entre a equipe técnica capacitada no modelo MR-MPS- } \\
\text { SW influenciou na mudança para o CMMI-DEV. }\end{array}$ & 1,84 \\
\hline Q25 & $\begin{array}{l}\text { O trabalho de melhoria do processo de software baseado no modelo } \\
\text { MR-MPS-SW aumentou substancialmente a competência da equipe } \\
\text { em engenharia de software. }\end{array}$ & 3,75 \\
\hline Q26 & $\begin{array}{l}\text { O trabalho de implantação e execução do modelo MR-MPS-SW } \\
\text { melhorou o desempenho global da organização. }\end{array}$ & 3,67 \\
\hline Q27 & $\begin{array}{l}\text { Durante a vigência do certificado do modelo MR-MPS-SW, os custos } \\
\text { com o desenvolvimento de software reduziram consideravelmente. }\end{array}$ & 2,59 \\
\hline Q28 & $\begin{array}{l}\text { Durante a vigência do certificado do modelo MR-MPS-SW, o tempo } \\
\text { do ciclo de desenvolvimento de software reduziu consideravelmente. }\end{array}$ & 2,50 \\
\hline Q29 & $\begin{array}{l}\text { Durante a vigência do certificado do modelo MR-MPS-SW, aumentou } \\
\text { consideravelmente a satisfação dos clientes. }\end{array}$ & 3,34 \\
\hline Q30 & $\begin{array}{l}\text { Foram estabelecidas metas claras para as atividades de melhoria do } \\
\text { processo de software pelo MR-MPS-SW na organização. }\end{array}$ & 3,84 \\
\hline Q31 & $\begin{array}{l}\text { Há um amplo entendimento dos objetivos e políticas da melhoria do } \\
\text { processo de software sugeridas pelo MR-MPS-SW. }\end{array}$ & 3,67 \\
\hline Q32 & $\begin{array}{l}\text { Os objetivos de implantação do modelo MR-MPS-SW estão } \\
\text { estreitamente relacionados com os objetivos de negócio da } \\
\text { organização. }\end{array}$ & 3,75 \\
\hline Q33 & $\begin{array}{l}\text { A organização tem um bom entendimento entre metas de curto prazo } \\
\text { e metas longo prazo estabelecidos pelo modelo MR-MPS-SW. }\end{array}$ & 3,50 \\
\hline Q34 & $\begin{array}{l}\text { É oferecido treinamento para desenvolver as habilidades e } \\
\text { conhecimentos necessários para realizar a implementação do MR- } \\
\text { MPS-SW. }\end{array}$ & 4,25 \\
\hline Q35 & $\begin{array}{l}\text { Os benefícios do modelo MR-MPS-SW são disseminados entre todos } \\
\text { envolvidos no processo de implantação da organização. }\end{array}$ & 3,92 \\
\hline
\end{tabular}

De acordo com os valores de RM das questões Q15 $(3,84)$ e Q16 $(4,09)$ verificou-se que as organizações estudadas pretendiam expandir seu mercado para outros países e que implementaram os modelos MR-MPS-SW e CMMI-DEV para aumentar as oportunidades de negócio dentro e fora do Brasil, apontando assim, indícios da influência dos fatores F16 (Internacionalização da organização) e F17 (Reconhecimento do modelo CMMI no exterior).

Os participantes concordaram que os objetivos da implantação do modelo MR-MPS-SW estavam estreitamente relacionados com os objetivos de negócio da organização (Q32, 
$\mathrm{RM}=3,75$ ), possibilitando confirmar que os fatores F1 (Estratégia de implantação da melhoria do processo de software) e F2 (Conciliação com os interesses da organização) não influenciaram na migração.

Os fatores F3, F5 e F9 relacionados a rotatividade e aos aspectos técnicos da equipe envolvida no processo de software não influenciaram na migração, analisados nas questões (Q24, Q25, Q31, Q33, Q34).

Observou-se nas respostas que após a implantação do MR-MPS-SW as empresas não reduziram os custos de desenvolvimento e também o tempo do ciclo de desenvolvimento de software (Q27, Q28) mas melhoraram o desempenho global, fato apontado pela questão Q26 $(\mathrm{RM}=3,60)$. Desse modo, houve influência moderada do fator F7 na migração para o CMMI.

O teste de hipótese aplicado para verificar a existência de semelhança entre as respostas (Q15 a Q35) dos participantes com o perfil de gerente e desenvolvedor, apontou que não houve diferença significativa $(p>0,05)$ para todas as questões, com exceção da questão Q17. O resultado mostra que os dois perfis compartilham da mesma visão sobre a aplicação dos processos de melhoria de desenvolvimento de software (MR-MPS-SW e CMMI-DEV). No entanto, a divergência de opiniões acerca da questão Q17 foi atribuída aos diferentes contextos organizacionais em que cada perfil estava inserido.

Na questão Q34 todos concordaram que não houve problemas no processo de implantação do modelo MR-MPS-SW e na capacitação técnica oferecida por uma consultoria especializada por meio de treinamentos e tutoria (mentoring).

De maneira geral, os resultados do questionário apontaram a influência dos fatores F14 (Exigência do cliente), F16 (Internacionalização da organização) e F17 (Reconhecimento do CMMI no exterior) na migração do modelo MR-MPS-SW para o CMMI-DEV.

\subsection{Entrevista aplicada as empresas que realizaram a migração}

A entrevista semiestruturada foi aplicada por um moderador a 10 participantes. Do total que respondeu a entrevista, dois registraram suas respostas por meio da voz, via internet, e os demais utilizaram um formulário eletrônico, o qual promoveu uma redução no número de abstenções.

$\mathrm{Na}$ entrevista foram encontradas novas evidências que apontaram a Adequação do modelo de SPI à realidade organizacional. Dentre as respostas, uma sugeriu que os modelos não deveriam ser monolíticos, e sim flexíveis, adequando-se à realidade da organização. Os participantes também mencionaram, como ponto positivo, a escalabilidade entre os níveis de maturidade do modelo brasileiro. Além disso, aportaram que os incentivos financeiros oferecidos pela Softex e o baixo custo de implantação do MR-MPS-SW motivaram as empresas de software a adotarem inicialmente este modelo. Estes fatores foram identificados por meio da ocorrência dos códigos "Fomento para implantação do MPS nos níveis iniciais de maturidade" e "Baixo custo de implantação do MPS.BR" apresentados na Figura 1. 
Figura 1 - Motivadores que influenciaram à adoção inicial do MPS.

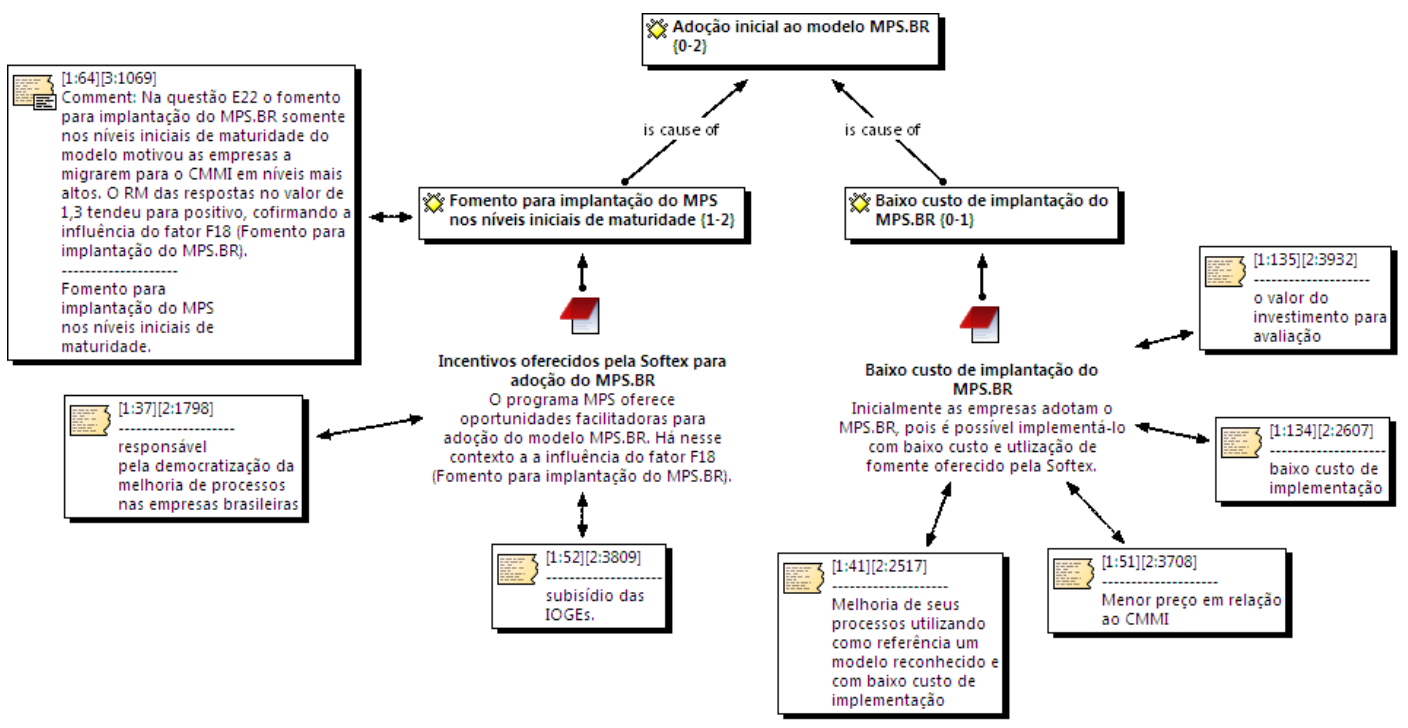

Os participantes informaram que o incentivo financeiro oferecido pela Softex somente nos níveis iniciais de implantação e avaliação do modelo nacional influencia de forma positiva a migração do MR-MPS-SW para o CMMI-DEV. Dessa forma, a influência do fator F18 (Fomento para a implantação do MR-MPS-SW) ocorreu apenas nas migrações para os níveis mais altos do CMMI-DEV, onde não há a presença de incentivos financeiros nos níveis compatíveis do MPS.

Os entrevistados reconheceram que os clientes passaram a exigir a certificação das organizações de software no CMMI-DEV devido ao alcance do modelo no exterior. Foi possível identificar a influência do fator F14 (Exigência do cliente) no processo de migração para o CMMI-DEV. As empresas também visualizavam na migração uma possibilidade de aumentar a quantidade de projetos de grande porte executados.

Nenhum entrevistado relatou problemas quanto ao relacionamento da equipe organizacional com a consultoria especializada do MR-MPS-SW. Desse modo, considerou-se que o fator $\mathrm{F} 4$ não motivou o processo de migração.

A respostas apontaram que os membros das organizações estavam motivados e satisfeitos com a adoção do MR-MPS-SW. Por isso, estabeleceu-se que os fatores F5 (Motivação e satisfação dos membros da organização) e F9 (Nível de comprometimento da equipe) não foram influenciadores no processo de migração das organizações de software.

Os entrevistados avaliaram negativamente o nível de internacionalização do MR-MPSSW. Relataram que o modelo MR-MPS-SW não possuía o nível de internacionalização desejado pelas organizações para que pudessem expandir o mercado e aumentar as oportunidades de negócio no exterior.

Os respondentes indicaram de forma positiva que as empresas tinham a necessidade de internacionalização. A Figura 2 expõe a associação causal entre os códigos "Necessidade de Internacionalização da organização" (15 citações) e "Internacionalização da Organização", que identificaram a influência do fator F16 (Internacionalização da organização) no processo de migração para o CMMI-DEV. Ainda na Figura 2, observase o código "Migração da certificação do modelo MPS.BR para o CMMI" que representa o processo de migração do MR-MPS-SW para o CMMI-DEV e que ocorre devido a 
presença dos códigos "Níveis de internacionalização do MPS.BR", "Marketing internacional da organização", "Internacionalização da organização", "Exigência de certificação do CMMI", "Reconhecimento do modelo CMMI" e "Investimento em níveis mais altos do MPS.BR é equivalente ao CMMI". Dessa forma, é possível apontar a influência na migração do fator F17 (Reconhecimento do modelo CMMI no exterior). Também foi possível verificar que o fator F14, representado pelo código "Exigência dos clientes internacionais e nacionais na certificação no CMMI" influenciou no processo de Internacionalização da organização.

Os entrevistados não apostaram como motivador da migração possíveis disputas políticas dentro da organização. Outro tema considerado foi a quantidade de processos presentes em cada nível de maturidade do MR-MPS-SW, que não foi aprovada por metade dos participantes. Os entrevistados informaram que a gerência de portfólio não está adequada ao nível F do MR-MPS-SW.

Figura 2 - Codificação do processo de migração do MPS para o CMMI

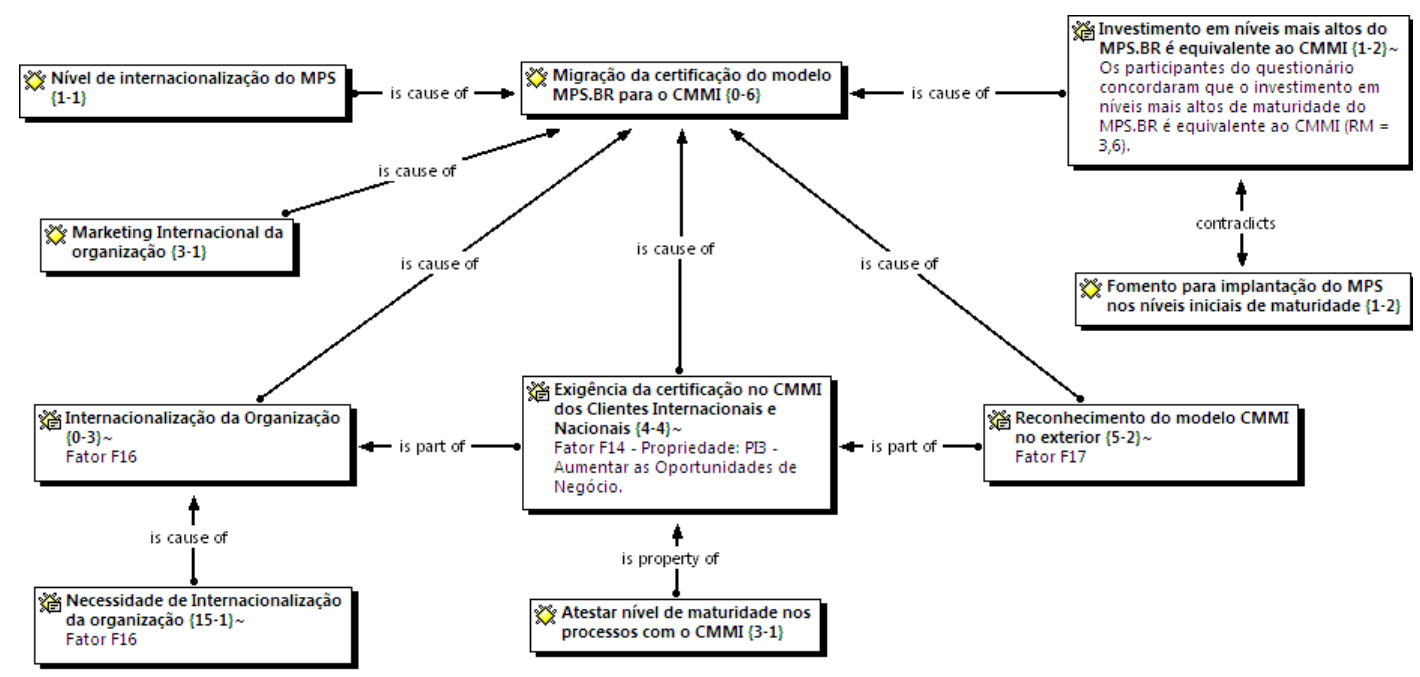

A Figura 3 mostra parte das 15 citações que serviram de base ao código "Necessidade de Internacionalização da organização", também presente na Figura 2. Foi possível identificar nas citações um relato sobre maior reconhecimento internacional da unidade organizacional avaliada no CMMI-DEV, o que reforçou a influência dos fatores F16 (Internacionalização da organização) e F17 (Reconhecimento do modelo CMMI no exterior), também codificados na Figura 18, no processo de migração 
Figura 3 - Códigos gerados na análise sobre a influência da Internacionalização na migração.

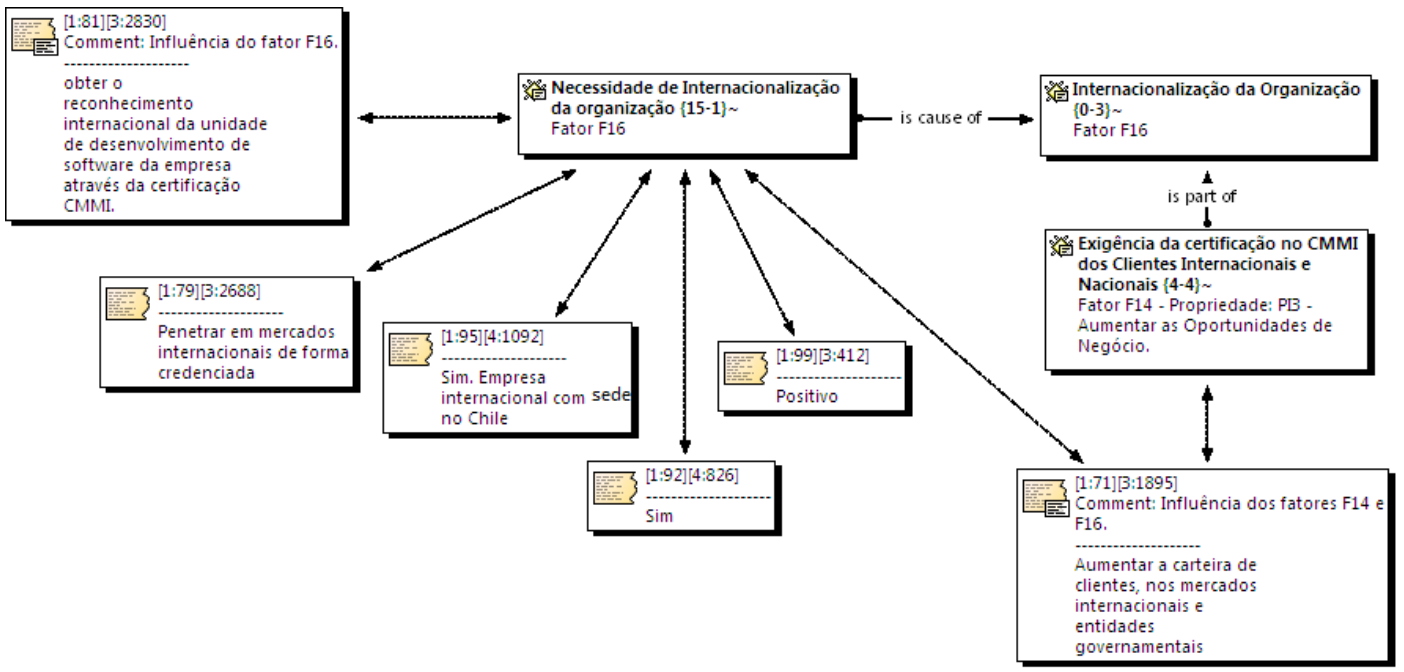

As respostas também reforçaram que após a certificação no CMMI-DEV as empresas obtiveram novos projetos nacionais e internacionais, pois eram consideradas mais maduras na execução de seus processos. Por isso, a certificação era exigida pelos clientes (F14) para atestar a maturidade na execução dos processos.

Como resposta à questão de pesquisa RQ3 (Quadro 15), as evidências observadas na entrevista confirmaram forte influência dos fatores Exigência do cliente (F14), Internacionalização da organização (F16) e Reconhecimento do modelo CMMI-DEV no exterior (F17) na migração. O fomento na implantação do MR-MPS-SW somente em níveis iniciais de maturidade (F18) e a otimização da monitoria e controle dos processos implantados (F7) contribuíram moderadamente na migração para o CMMI. Além disso, não apresentaram influência na migração para o CMMI-DEV os seguintes fatores: Estratégia de implantação da melhoria do processo de software (F1), Conciliação de interesses da organização (F2), Competência dos membros da organização de software (F3), Respeito da consultoria pela organização de software (F4), Motivação e satisfação dos membros da organização (F5), Metodologia formal da implantação (F6), Falta de competência da consultoria especializada (F8), Nível de comprometimento da equipe (F9), disputas políticas dentro as organização avaliada pelo MPS.BR (F10). A influência de cada fator na migração do MR-MPS-SW para o CMMI-DEV foi resumida no Quadro 4.

Quadro 4 - Influência dos fatores analisados na pesquisa.

\begin{tabular}{|l|l|c|l|}
\hline ID & Fatores & $\begin{array}{l}\text { Influenciaram } \\
\text { na migração }\end{array}$ & $\begin{array}{l}\text { Não influenciaram } \\
\text { na migração }\end{array}$ \\
\hline F14 & Exigência do cliente & $\bullet$ & \\
\hline F16 & Internacionalização da organização. & $\bullet$ & \\
\hline F17 & $\begin{array}{l}\text { Reconhecimento do CMMI no } \\
\text { exterior. }\end{array}$ & $\bullet$ & \\
\hline F18 & Fomento na implantação do MPS. & $\bullet$ & \\
\hline
\end{tabular}




\begin{tabular}{|c|c|c|c|}
\hline ID & Fatores & $\begin{array}{l}\text { Influenciaram } \\
\text { na migração }\end{array}$ & $\begin{array}{l}\text { Não influenciaram } \\
\text { na migração }\end{array}$ \\
\hline F7 & Monitorar e controlar os processos. & - & \\
\hline F1 & Estratégia de implantação de SPI. & & $\bullet$ \\
\hline $\mathrm{F} 2$ & $\begin{array}{l}\text { Conciliação com os interesses da } \\
\text { organização. }\end{array}$ & & • \\
\hline F3 & $\begin{array}{l}\text { Competência dos membros da } \\
\text { organização. }\end{array}$ & & $\bullet$ \\
\hline $\mathrm{F} 4$ & Respeito da consultoria. & & $\bullet$ \\
\hline F5 & $\begin{array}{l}\text { Motivação e satisfação dos } \\
\text { membros da organização. }\end{array}$ & & - \\
\hline F6 & $\begin{array}{l}\text { Metodologia formal da implantação } \\
\text { do modelo. }\end{array}$ & & $\bullet$ \\
\hline F8 & $\begin{array}{l}\text { Falta de competência da consultoria } \\
\text { especializada }\end{array}$ & & • \\
\hline F9 & $\begin{array}{l}\text { Nível de comprometimento da } \\
\text { equipe. }\end{array}$ & & $\bullet$ \\
\hline F10 & $\begin{array}{l}\text { Disputas política dentro da } \\
\text { organização. }\end{array}$ & & $\bullet$ \\
\hline
\end{tabular}

\section{Conclusão}

O estudo concluiu que o modelo MR-MPS-SW é capaz de atender e se adequar às necessidades das empresas de software do mercado nacional. No entanto, a maioria das organizações pretendia expandir seus produtos ou serviços para o mercado externo, e também aumentar o número de clientes nacionais, mas os mesmos exigiam a avaliação de seus processos no CMMI. Por isso, foram encontradas evidências da migração da avaliação dos níveis iniciais de maturidade do MR-MPS-SW para os níveis equivalentes ou mais altos de maturidade do CMMI-DEV, principalmente nas empresas de médio porte e que possuíam uma equipe técnica capacitada para execução dos processos exigidos pelo MPS.

Fatores como a exigência dos clientes nacionais e internacionais, o reconhecimento internacional do CMMI, a internacionalização da organização e o marketing dos produtos e serviços foram os principais motivadores da migração. $O$ fomento na implantação do MPS somente em níveis iniciais de maturidade e a otimização da monitoria e controle dos processos implantados contribuíram moderadamente na migração para o CMMI. Os fatores relacionados a aspectos técnicos da equipe, políticos da organização e da implantação do modelo não foram apontados como motivadores da migração. Além disso, ficou evidente a competência das equipes de implementação e avaliação do modelo MPS que demonstra uma boa capacidade operacional de expansão do modelo. 
Como contribuição, os métodos e instrumentos definidos, bem como os fatores influenciadores da migração identificados por esta pesquisa poderão ser utilizados pela Softex na avaliação anual do modelo (iMPS) aplicada em todas empresas avaliadas no MPS. Com base nos índices coletados, o órgão poderá estabelecer um conjunto de políticas de continuidade, com o objetivo de manter as organizações avaliadas associadas ao programa. A pesquisa contribuiu também para reduzir a lacuna existente na literatura acerca da investigação dos fatores críticos de sucesso na melhoria do processo de software definida pelo modelo MR-MPS-SW sobre a perspectiva de implantação e avaliação do modelo, e, principalmente, da sua continuidade.

\section{References}

Almeida, C. D. A. Continuidade da Execução dos Processos de Software em Empresas Avaliadas no MPS.BR. Dissertação de mestrado. Universidade de Fortaleza, 2011.

Dyba, T. An Instrument for Measuring the Key Factors of Success in Software Process Improvement. Empirical Software Engineering, 2000.

Fuggetta, A. Software Process: A Roadmap. Proceedings of the Conference on The Future of Software Engineering, 2000.

Glaser, B.G.; Strauss, A. The Discovery of Grounded Theory: Strategies for Qualitative. 1967.

ISO - International Organization for Standardization. ISO/IEC 12207 Systems and software engineeringv- Software life cycle processes. Geneve, 2008.

ISO - International Organization for Standardization. ISO/IEC 15504-2: Information Technology - Process Assessment - Part 2 - Performing an Assessment. Geneve, 2003.

Kitchenham, B.; Pfleeger, S. L. Guide to Advanced Empirical Software Engineering. Springer London, 2008.

Likert, R. A Technique for the Measurement of Attitudes. Archives of Psychology, 1932.

Marconi, M. E.; Lakatos, E. M. Fundamentos de metodologia científica. $5^{\text {a }}$ Ed. São Paulo: Atlas, 2003.

Montoni, M. A. Uma Investigação sobre os Fatores Críticos de Sucesso em Iniciativas de Melhoria de Processos de Software. Tese de doutorado. Universidade Federal do Rio de Janeiro/ Instituto Alberto Luiz Coimbra de Pós-Graduação e Pesquisa de Engenharia - UFRJ/COPPE, 2010.

Noblitt, G. W.; Hare, R. D. Meta-Ethnography: Synthesizing Qualitative Studies (Qualitative Research Methods). Sage Publications Ltd, 1988.

Oliveira, L. H. Exemplo de cálculo de Ranking Médio para Likert. Notas de Aula. Metodologia Científica e Técnicas de Pesquisa em Administração. Mestrado em Adm. e Desenvolvimento Organizacional. PPGA CNEC/FACECA: Varginha, 2005.

Santos, G., Kalinowski, M., Rocha, A. R., Travassos, G. H., Weber, K. C., Antonioni, J. A. MPS.BR: A Tale of Software Process Improvement and Performance Results in the Brazilian Software Industry. In Proceedings of Seventh International Conference on the Quality of Information and Communications Technology, 2010. 
SEI - Software Engineering Institute. CMMI for Development, Version 1.3. Technical Report CMU/SEI-2010-TR-033. Software Engineering Institute - Carnegie Mellon University, 2010.

Singer, J.; Sim, S. E.; Lethbridge, T. C. Software Engineering Data Collection for Field Studies. Selecting Empirical Methods for Software Engineering Research, 2008.

Softex. Guia Geral MPS de Software. Associação para Promoção da Excelência do Software Brasileiro - Softex, 2012a.

Strauss, A., Corbin, J. Basics of Qualitative Research: Techniques and Procedures for Developing Grounded Theory. $2^{\mathrm{a}}$ Ed. SAGE Publications, 1998.

Travassos, G. H. iMPS 2011: resultados de desempenho das empresas que adotaram o modelo MPS de 2008 a 2011. SOFTEX, 2012.

Travassos, G. H., Kalinowski, M. iMPS 2012 - Evidências Sobre o Desempenho das Empresas que Adotaram o Modelo MPS-SW desde 2008. Softex, 2013.

Unterkalmsteiner, M.; Islam, M.; Cheng, C. K.; Permadi, R. B.; Feldt, R. Evaluation and Measument of Software Process Improvement: A Systematic Review. IEEE Transactions on Software Engineering, 2012.

Weber, K. C.; Araújo E.; Machado, C. A. F.; Scalet, D.; Salviano, C. F.; Rocha, A. R. C. Modelo de Referência e Método de Avaliação para Melhoria de Processo de Software - versão 1.0 (MR-MPS e MA-MPS). IV Simpósio Brasileiro de Qualidade de Software, 2005b. 